\title{
Applications of Microalgae as Feed Additives in Aquaculture
}

\author{
Jinghui $\mathrm{LI}^{\mathrm{a}}$, Ze FAN ${ }^{\mathrm{b}}, \mathrm{Mu} \mathrm{QU}^{\mathrm{c}}$, Xiuting QIAO ${ }^{\mathrm{d}}$, Jinhui SUN ${ }^{\mathrm{e}}$, Dongqing BAI ${ }^{\mathrm{f}}$ and \\ Zhenyan $\mathrm{CHENG}^{\mathrm{g}^{*}}$
}

\author{
Tianjin Key Lab of Aqua-Ecology and Aquaculture, College of Fisheries, Tianjin Agricultural \\ University, Tianjin 300384, P.R. China.

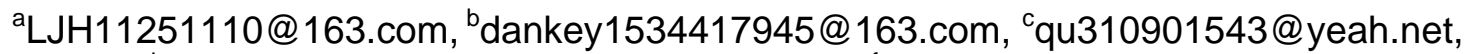 \\ dqxt65@sohu.com, ejhsun1008@163.com, 'baidongqing@tjau.edu.cn, \\ gchengzhenyan2005@126.com,
}

\section{Keywords: Microalgae; Aquaculture; Feed additive; Application}

Abstract. Microalgae are a group of small size and lower plants that can conduct photosynthesis, which are characterized by riching in protein, fat (polyunsaturated fatty acids), polysaccharides, vitamins, antioxidants, pigments, trace elements, etc. Its nutritional value can be comparable to meat, eggs, and even better than the food. Many studies demonstrated that microalgae could be used as feed additives to help promote the growth of aquatic animals and improve their immunity effect in aquaculture and so on. In this paper, the main physiological function of microalgae as well as the applications in aquaculture were reviewed. Hopefully, which could provide some reference value for microalgae used in aquaculture.

\section{Introduction}

With the development of aquaculture, the needs of bulk feed materials, such as for soybean meal, fish meal and other resources, are constantly going up and prices are increasing every year, so the research for seek new sources of raw materials has been a focus. Microalgae has many advantages (rich in a variety of important bioactive substance-protein, amino acids, polysaccharides, unsaturated fatty acids and cytochrome etc.), which make it become a new source of feed material (source of protein, fatty acids, vitamins, etc.). And, along with the concepts of healthy farming and food safety receive increasing attention in aquaculture, the entire aquaculture industry is gradually changing from the simple to increase production to obtain benefits, to improve product quality (safety and health, taste, color, nutrients, etc. ) to get benefits. Thus, the advantages of microalgae appear increasingly in aquaculture industry.

In recent years, with the microalgae cultivation technology advances, particularly the research of through heterotrophic fermentation, you can get $50 \mathrm{~g} / \mathrm{L}$ or so chlorella powder at a $5 \mathrm{t}$ fermentation tank through training in $3 \mathrm{~d}$, which greatly reduce the cost. Therefore, the use of microalgae as feed additives is more and more widely in aquaculture.

\section{The Main Physiological Function of Microalgae as a Feed Additive}

Microalgae are Rich in Protein, with Growth-promoting Effect. The section headings are in boldface capital and lowercase letters. Second level headings are typed as part of the succeeding paragraph (like the subsection heading of this paragraph).Microalgae are rich in nutrients. Its protein content can be as high as $50 \%$, for example dried spirulina contain protein up to $60 \% \sim 90 \%$. It also contains all the essential amino acids required for animals, especially riching in lysine and threonine which cereals are lack of it. Adding microalgae to feed both can play "complementary proteins", and can also solve the problem that cereals' protein nutritional value is low. As feed or feed additives of fish, shrimp, crab, shellfish and sea cucumber, microalgae are widely used in aquaculture. A trial was designed to study the effects of adding algae powder to the basic diet of bluntnose black bream to

replace fishmeal on growth and gene expression of related growth metabolism in Megalobrama amblycephala ${ }^{[1]}$. The results showed that the growth hormone gene expression of dosage $1.5 \%$ and $3 \%$ treatment group was significantly higher than the other treatment groups. The growth rate, 
specific growth rate and protein efficiency of 3\% dosage treatment group was significantly higher than control group. A similar trial was conducted in sea cucumber ${ }^{[2]}$. Its results showed that the weight gain rate and specific growth rate reach the maximum in group of $25 \%$ replaced. Some trials were conducted to study the effects of Spirulina on growth performance and the resistance immunity and disease in shrimp ${ }^{[3-5]}$. The results showed that the right amount of spirulina could promote growth, improve digestion and utilization of feed, enhance the resistance immunity and disease and improve survival rate of shrimp. Similar trials were conducted to study the effects of Spirulina on growth-promoting properties in pengze carp ${ }^{[6]}$ and abalone ${ }^{[7]}$. The results showed that significantly improving were observed in growth performances and feed conversion in the groups of appropriate adding amount. A trial was conducted to add the right amount of spirulina powder in the bait of $\mathrm{crab}^{[8]}$. At the end of the nursery, nursery time of the test group than the control group shortened three days and the survival rate increased $14.2 \%$, significantly saving nursery costs.

Rich in cytochrome of microalgae, with the effect of hyperchromic. Microalgae are rich in $\beta$-carotene, astaxanthin and lutein. It can improve the animal's color, especially the color of ornamental fish. Some studies show that adding spirulina to the feed can effectively improve the body color of fish. The floating bait with contain spirulina for the goldfish, koi and tropical fish etc. of ornamental value have been produced in Japan. Not only the growth were promoted, but also the vividness of color markings of the fish were increased. It is very popular in the international market ${ }^{[9]}$. A trial ${ }^{[10]}$ was conducted by feeding Regalecus glesne with the addition of $5 \%$ and $10 \%$ of spirulina powder. The results showed that the pigment content of the experimental fishes' skin with feeding the spirulina powder group were significantly higher than the control group with feeding sardines after $83 \mathrm{~d}$. And with its appearance is bright and green the yellow is bright ang body color has been clearly improved. Similar results were found in koi $^{[11-12]}$ and goldfish ${ }^{[13]}$.

Rich in polysaccharides of microalgae, enhancing immunity. Microalgae are rich in polysaccharides, which can improve animal immunity. A trial was conducted to study the effects of spirulina powder on immunity in silver carp ${ }^{[14]}$. The results showed that the activity of superoxide dismutase and lysozyme of serum were increased. Some trials were conducted to study the effects of seaweed polysaccharide on immunity in Chinese shrimp ${ }^{[15]}$ and scallop ${ }^{[16]}$. The results showed that the immune function were improved. Another study proved that seaweed polysaccharides could activate kidney cells in the front and white blood cells active and enhancement the resistance on Aeromonas hydrophila sp. of Lanmaozulu. Some studies in vitro showed that polysaccharide, isolated from marine microalgae Dinoflagellate gymnodinium sp.A3 and Cochlodimium polykrikoides respectively, could inhibit telomerase activity of K562 cells with $10 \mu \mathrm{g} / \mathrm{L}$ concentration and could completely inhibit enveloped viruses invading the host in vitro. Some studies showed that $200 \mathrm{mg} / \mathrm{kg}$ of spirulina polysaccharide significantly inhibited the proliferation of mice ascites hepatoma cells. Within 3 24h its inhibition of DNA, RNA and protein of S180 and ascites hepatoma cells were enhanced with prolonging duration of action. Its inhibition of cancer DNA synthesis, is a type of DNA metabolism disturbance. Although spirulina polysaccharide don't directly kill cancer cells, it can inhibit cancer cell proliferation by enhancing the body's immune system.

Rich in polyunsaturated fatty acids of microalgae, enhancing the value of aquatic products. Microalgae are rich in eicosapentaenoic acid (EPA) and docosahexaenoic acid (DHA). So it could improve the growth and reproduction, immunity and nutritional value of the aquatic. Some trials were conducted to study the effects of microalgae on growth, DHA and EPA in juvenile flounder ${ }^{[19-20]}$. The results show that EPA, DHA and the ratio of n-3/n- 6 were improved. The results also showed that FCR and growth performance were promoted by microalgae. Thus, it can be deduced that microalgae effectively improve the health value of flounder.

Other active ingredients with special functions. In recent years, in the study of chlorella, special functional ingredients-Chlorella Growth Factor(CGF) were found, which aren't available in other microalgae. Its main component is a short-chain polypeptide protein and nucleic acid the complex. It contains 17 kinds of amino acids, of which there are nine kinds of essential functional amino acids. CGF play an important role in promoting cell division, anti-cancer, anti-radiation, blood pressure and improve animal immunity aspects ${ }^{[21]}$. Other active ingredients, such as dimer acid and spermine, etc., 
were also contained in microalgae. The physiological functions of fish, such as strengthening the secretion of the digestive yeast of larval and regulating digestive enzyme activity, were regulated by them. Besides, to adjust the release of pancreas digestive enzymes, the cholecystokinin were producted by the stimulation of poly ugly amine. Spermine could improve coating enzyme (alkaline phosphatase and aminopeptidase) activity of the intestines and promote mature of intestinal epithelium of larvae. In addition, microalgae can also adjust the growth of beneficial bacteria, antimicrobial activity, and inhibit the eruption of opportunistic pathogen in aquaculture water to maintain the microecosystem balance of culture water, live bait and intestinal of larvae ${ }^{[22-24]}$. Microalgae contain a variety of antioxidants (such as multi-carotenoids, vitamins) as well as some special phytochemicals. Although the mechanism has not been fully elucidated, it has been found that there were a certain effect on aquatic animals.

\section{The specific applications of microalgae on aquaculture}

Living microalgae as a bait. Currently, microalgae are still directly used to feed animals and enhance animal feed in aquaculture feed. For example, fortifing rotifers that were feed by bread yeast culture with algae of enriching with DHA, which can greatly improve its nutritional value. The applications of microalgae in fish, shellfish, crustaceans nursery has reached more than forty kinds in abroad. In the 1950s, the culture of marine microalgae biological bait began. There have been more than twenty kinds, such as Phaeodactylum tricornutum Bohlin, Nitzschiaclosteriumfminu- tissima, Chaetoceros muelleri, Chaetocerosgracilis, Skeletonema costatum (Greville) Cleve, Platymonas subcordiformis , Pyramidomonas sp., Dunaliella salina, Chlorella, Nannochloropsis oculata, Lsochrysis, Lsochrysis zhanjiangensis, Spirulina platensis and so on, were cultured. The Nannochloropsis oculata and Isochrysis galbana are applied at most in strengthening of initial feeding, as these two algae are rich in DHA and EPA. Because of the different nutrients of microalgae and different nutrients need of aquatic animals, different baits were used to culture different aquatic animals and different developmental stages.

Storaging microalgae as a bait. The living microalgae are necessary bait for many larvae aquatic animals. The quality of microalgae are directly related to the success of the nursery, but the cultivation of living microalgae are greatly affected by the environment conditions. Therefore, to ensure the quantity and quality of bait, storage microalgae can be used to instead of live microalgae. The methods of storage bait are freeze-drying, frozen (without protective agent or protective agents added), refrigerated, spray drying, after centrifugation adding preservatives to form alginate paste and so on. Among them, frozen (with protective agent) for one month, the survival rate was $20 \%$. After saving a month under $4{ }^{\circ} \mathrm{C}$, the survival rate was $80 \%$.

Strengthening rotifers, artemia with microalgae. At present, microalgae were often used as bait in artificial breeding of shellfish. The nauplii of rotifers, artemia were used as bait in nursery production and the adult of copepods and artemia as bait in early cultivation in fish and shrimp. However, due to the restrictions of conditions and costs, fresh yeast are usually used to culture rotifers, artemia, copepods etc., which are used for fish and shrimp nursery. They are lack of essential highly unsaturated fatty acids, for those with yeast production animal feed, must use highly unsaturated fatty acid-rich plant food to strengthen for some time before feeding the fish and shrimp. The rotifers, Artemia, copepods, etc., which are strengthened or directly cultured with these high-fat microalgae, can not only improve effec of feedt, but also save microalgae, shorten the time to strengthen cultivation and decrease costs.

Adding microalgae to feed by powder. Screening the microalgae that can be used as feed additives, then classifing it as functions: rich in proteins, polysaccharides, fatty acids and cytochrome (chlorophyll, astaxanthin, and lutein). Pick out the microalgae rich in protein (such as Spirulina platensis), rich in unsaturated fatty acids (such as Schizochytrium and Nannochloropsis oculata) and rich in pigment cells(such as Spirulina). Culture the screening microalgae by large-scale to get microalgae powder. Adding it to the feed of different proportions to study the effect and regulation mechanism of specific microalgae on body color, growth, immunity, meat quality, et al.. According 
to the need of animals, the conditions of storage were changed to be suitable for the needs of farmed animals. For example, some certain chlorella can turn red or orange and accumulate large amounts of carotenoids and astaxanthin in the conditions of nitrogen deficiency or high salt. So this microalgae can be addde to bait of aquatic animals to make them color more vivid ${ }^{[25]}$.

\section{Conclusions}

The advantages of microalgae in promoting growth and improving survival have been proven, and it can not be replaced by other artificial bait. There are quite a lot of advantages and huge market of microalgae for aquaculture, which is destined to be a more wide market in the near future.

\section{Acknowledgements}

This work was supported by Tianjin Innovative Research Team (TD12-5018), the key technologies R\& D program of Tianjin (13ZCZDNC00900) and Tianjin Research Program of Application Foundation and Advanced Technology (14JCQNJC15100). This study was also supported by National Natural Science Foundation of China (31402313).

\section{References}

[1] Lining Ci: Effect of the Diets Replacing Different Proportions of Fish Meal with Aglgae Powder on the Growth Performance, Nutrients Digestbility and Immunity of Bluntnose Black Bream (megalobrama amblycephala). Master thesis: Nanjing Agricultural College, 2011. [In Chinese]

[2] Xiaoying Tan, Zhi Luo, Xiaodong Li, et al.: Journal of Dalian Fisheries College. Forum Vol. 24 (2009), p. 559-562. [In Chinese]

[3] Jinxing He, Yan Dou, Jianqing Tang, et al.: Jiangsu Agricultural Sciences. Forum Vol. 2 (2009), p. 209-211. [In Chinese]

[4] Lihe Liu, Shixuan Zheng, Huanxin Xu, et al.: Journal of Fisheries. Forum Vol. 29 (2005), p. 791-797. [In Chinese]

[5] Fuli Zhang: Scientific fish. Forum Vol. 4 (1994), p. 25. [In Chinese]

[6] Huazhong Liu, Dingzhong Liu, Maozhong Yao, et al.: Fishery Sciences. Forum Vol. 3 (2004), p. 15-25. [In Chinese]

[7] Quanzhen Chen, Jiangning Ceng, Yibao Liao, et al.: Aquatic Science and Technology Information. Forum Vol. 31 (2004), p. 3-6. [In Chinese]

[8] Weiping Dai, Jianhong Gu and Xinming Yang: Freshwater Fisheries. Forum Vol. 30 (2000), p. 28-29. [In Chinese]

[9] Zhiqiong Li, Kailiu Wang and Zongxian Du: Feed Research. Forum Vol. 1 (2001), p. 15-18. [In Chinese]

[10] S. Okada, W. Liao, T. Mori, et al.: Nippon Suisan Gakkaishi. Forum Vol. 57 (1991), p. 1403-1406.

[11] Peimin He, Yinjiang Zhang and Wenhui He: Journal of Fisheries. Forum Vol. 23 (1999), p. 162-168. [In Chinese]

[12] Feiyu Yuan, XiaoBo Yang, Chenghong Liu, et al.: Reservoir Fisheries. Forum Vol. 1 (2003), p. 41-42. [In Chinese]

[13] Lei Xu: Journal of Hannan University. Forum Vol. 17 (1999), p. 366-368. [In Chinese] 
[14] Chunfang Cai, Xuehong Song, Xingfa Pan, et al.: Reservoir Fisheries. Forum Vol. 3 (2002), p. 20-22. [In Chinese]

[15] Shuqing Liu, Xiaolu Jiang, Haijing Mou, et al.: Ocean and Limnology. Forum Vol. 30 (1999), p. 278-283. [In Chinese]

[16] Haijin Mou, Xiaolu Jiang, Shuqing Liu,et al.: Journal of Qingdao Ocean University. Forum Vol. 29 (1999), p. 463-468. [In Chinese]

[17] K. Sogawa, T. Sumida, H. Hamakawa, et al.: Research Communications in Molecular Pathology and Pharmacology. Forum Vol. 99 (1998), p. 259-265.

[18] M. Hasui, M. Matsuda, K. Okutani, et al.: Int J Biol Macromol. Forum Vol. 17 (1995), p. 293-297.

[19] Min Xue, Aijie Li and Xianjuan Zhang: Forum Vol. 3 (2004). [In Chinese]

[20] Hongjin Qiao, Zhidong Song, Jingjing Ma, et al.: Effect of the Diets Replacing Different Proportions of Fish Meal with Algae Powder on the Growth Performance, Body Composition and Fatty Acid Composition of Juvenile Paralichthys olivaceus. The $9^{\text {th }}$ symposium of World Chinese fish and shrimp nutrition. 2012, Xiamen. [In Chinese]

[21] Hai Yan and Chunhua Yin: Prospects of Microalgae as Feed Additives. [In Chinese]

[22] K. Hjelmeland, B.H. Pedersen and E.M. Nilssen: Marine Biology. Forum Vol. 98 (1988), p. 331-335.

[23] J.P. Lazo, M.T. Dinis, G.J. Holt, et al.: Aquaculture. Forum Vol. 188 (2000), p. 339-351.

[24] A. Peres, C.L. Cahu and J.L. Zambonino Infante: Fish Physiology and Biochemistry. Forum Vol. 16 (1997), p. 479-485.

[25] T. Sano: Science of Algae. 1993, p. 100-108. 